\title{
Normal ranges of modified axial lead system electrocardiogram parameters
}

\author{
Peter W. Macfarlane, A. R. Lorimer, and T. D. V. Lawrie \\ From the University Department of Medical Cardiology, Royal Infirmary, Glasgow
}

The normal ranges of 3 orthogonal lead electrocardiogram parameters, derived from the modified axial lead system, have been determined by computer analysis. A discussion of the clinically significant parameters is presented, and for those who may not wish to use the modified version of the axial system the calculation of ranges for the original axial system is detailed in an Appendix.

A modification to the axial orthogonal lead electrocardiogram recording system of $\mathrm{McFee}$ and Parungao (196I) has recently been described (Macfarlane, 1969). In order to determine the normal ranges of parameters for the modified system a group of normal subjects had 3 lead electrocardiograms analysed and interpreted by computer. This paper describes the results obtained.

\section{Methods}

Two hundred and sixty-five normal male and female subjects whose ages ranged from 4 to 80 years were studied. Their age distribution stratified in three groups, up to 14 years, 15 to 29 years, 30 years and over, is shown in Table 1 . Each patient was assessed as normal on the basis of physical, radiological, and conventional 12 lead electrocardiographic examination.

Three lead electrocardiograms were tape-recorded using standard FM techniques and replayed to a PDP-8 computer for analogue to

TABLE I Age distribution of 265 subjects used in the study

\begin{tabular}{cc} 
Age-group & Total \\
\hline$\leq 14$ & 18 \\
$15 \rightarrow 29$ & 94 \\
$\geq 30$ & 153 \\
\hline & 265
\end{tabular}

digital conversion. Data were subsequently transferred to a $\mathrm{KDF}_{9}$ computer for further analysis. Approximate wave onset and termination points were obtained using the concept of spatial velocity (Stallmann and Pipberger, 1961). Thereafter the true onset and termination were found for each lead by a scalar analysis of each lead individually. The onset of the QRS complex in each lead was taken as the baseline or zero value for the whole P-QRS-T complex. The method of wave recognition will be presented elsewhere in detail (Macfarlane, 197I).

All wave measurements were output to magnetic tape for analysis by a second programme which calculated means, standard deviations, and 96 per cent ranges for each parameter. If the value of a parameter was zero or did not exist - for example, an $R / Q$ ratio when there was no $Q$ wave, the parameter was not included in the estimation of the mean and standard deviation. Ninety-six per cent ranges were calculated, since it is well known that values of electrocardiogram parameters are not distributed normally (Simonson, 196I). Hence it is not correct to consider the range of a parameter to be the mean $\pm 2 \times$ standard deviation. In the case of the under-15 age-group with only 18 patients, one value of a parameter was removed from the lower and upper end of the range.

FIG. I The 3 orthogonal lead reference frame used. Lead $Z$ is directed positively to the anterior.
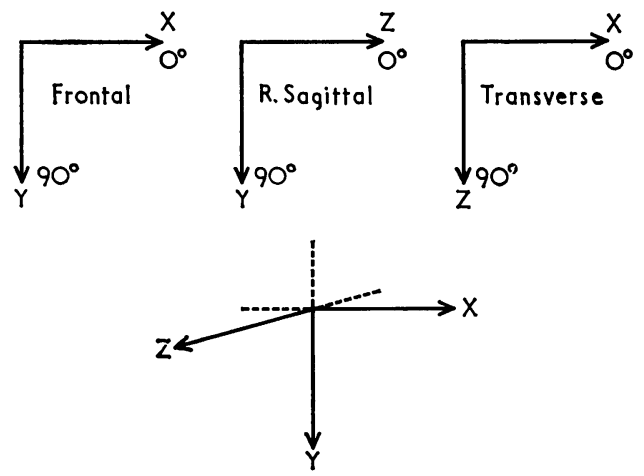
As a supplement, these ranges were studied to determine those which best separated normal from abnormal as discussed below. This led to the formation of diagnostic criteria which formed the basis of a computer programme for electrocardiogram interpretation.

\section{Electrocardiogram parameters}

Fig. I shows the co-ordinate frame of reference used. Lead $Z$ is directed anteriorly contrary to the recommendations of the American Heart Association Committee on Electrocardiography (1967). This was thought advisable to enable a straightforward comparison to be made with the praecordial leads VI, V2. In addition, with the choice of right sagittal projection as opposed to

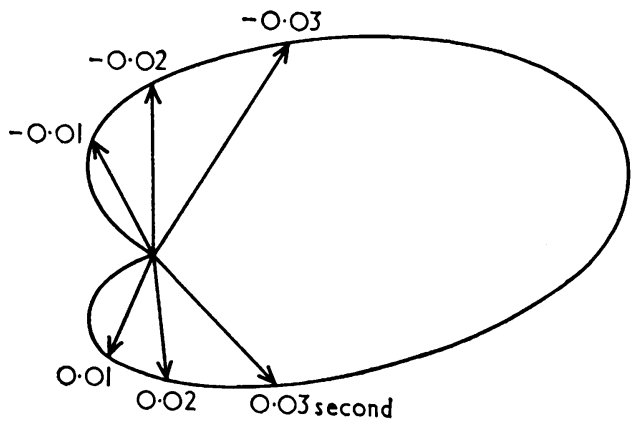

FIG. 2 Illustration of the $0.01,0.02,0.03$ sec. vectors after $Q R S$ onset and -0.03 , $-0.02,-0.01$ sec. vectors before $Q R S$ termination.

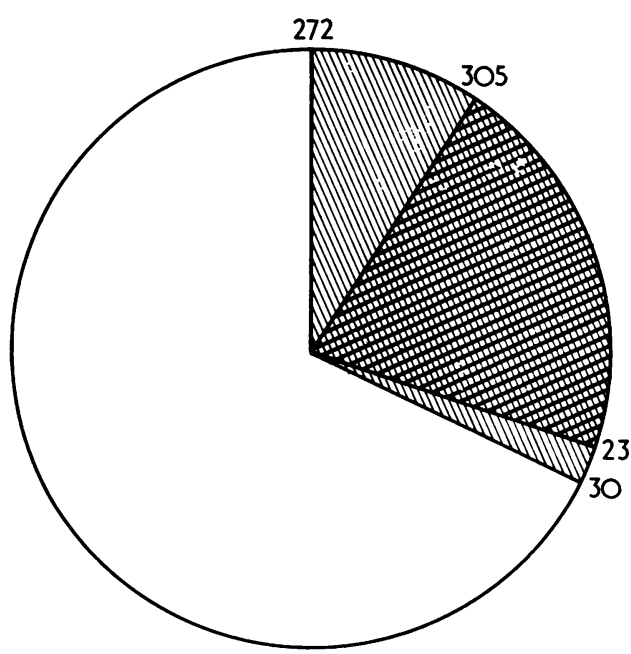

 20 Maximum spatial vector

FIG. 3 Ranges of normal values of the maximum vector in the transverse plane and the projection of the maximum spatial vector on to the transverse plane. left, the angular frame of reference was the same for each plane, frontal, right sagittal, and transverse. Angles were measured from $0^{\circ} \rightarrow 360^{\circ}$ in a clockwise direction (Fig. I).

All standard scalar wave amplitudes and duration measurements were obtained. Only $Q, R$, and $S$ wave durations are presented, since $P R, Q R S$, and QT intervals correspond with 12 lead measurements. In addition time-normalized data (Draper et al., 1964) were obtained for QRS and ST-T segments (see Fig. 4). In this technique the QRS or ST-T segment is divided into 8 equal time intervals and the wave amplitude determined at the end of each.

TABLE 2 Normal ranges of $P, Q, R, S, S T$ junction, and $T$ wave amplitudes: (a) denotes the under - I5 age-group; (b) denotes the 15 to 29 age-group; (c) denotes the over -29 agegroup. Results are expressed as mean \pm standard deviation with the 96 per cent range given below

\begin{tabular}{|c|c|c|c|c|}
\hline \multirow[t]{2}{*}{ Wave } & & \multicolumn{3}{|l|}{ Lead } \\
\hline & & $X$ & $Y$ & $Z$ \\
\hline $\mathbf{P}$ & $\begin{array}{l}\text { (a) } \\
\text { (b) } \\
\text { (c) }\end{array}$ & $\begin{aligned} & 0.07 \pm 0.05 \mathrm{mV} \\
&-0.03 \rightarrow 0.13 \rightarrow 0.06 \pm 0.04 \\
& 0.0 .03 \rightarrow 0.14 \\
& 0.07 \pm 0.04 \\
&-0.04 \rightarrow 0.13\end{aligned}$ & $\begin{array}{r}0.08 \pm 0.09 \\
-0.06 \rightarrow 0.15 \\
0.10 \pm 0.08 \\
-0.09 \rightarrow 0.25 \\
0.12 \pm 0.07 \\
-0.65 \rightarrow 0.27\end{array}$ & $\begin{array}{r}0.03 \pm 0.05 \\
-0.05 \rightarrow 0.09 \\
0.03 \pm 0.04 \\
-0.05 \rightarrow 0.11 \\
0.01 \pm 0.05 \\
-0.08 \rightarrow 0.09\end{array}$ \\
\hline Q & $\begin{array}{l}\text { (a) } \\
\text { (b) } \\
\text { (c) }\end{array}$ & $\begin{array}{l}0.13 \pm 0.09 \\
0.04 \rightarrow 0.27 \\
0.10 \pm 0.07 \\
0.03 \rightarrow 0.22 \\
0.09 \pm 0.07 \\
0.03 \rightarrow 0.27\end{array}$ & $\begin{array}{l}0.13 \pm 0.08 \\
0.05 \rightarrow 0.27 \\
0.10 \pm 0.07 \\
0.03 \rightarrow 0.26 \\
0.09 \pm 0.07 \\
0.03 \rightarrow 0.19\end{array}$ & $\begin{array}{l}- \\
-\end{array}$ \\
\hline $\mathbf{R}$ & $\begin{array}{l}\text { (a) } \\
\text { (b) } \\
\text { (c) }\end{array}$ & $\begin{array}{l}I \cdot 24 \pm 0.46 \\
0.65 \rightarrow 2.03 \\
I \cdot 08 \pm 0.33 \\
0.47 \rightarrow I .93 \\
I \cdot 07 \pm 0.35 \\
0.50 \rightarrow I \cdot 77\end{array}$ & $\begin{array}{l}I \cdot 20 \pm 0.46 \\
0 \cdot 60 \rightarrow I \cdot 72 \\
I \cdot 16 \pm 0 \cdot 47 \\
0.49 \rightarrow 2 \cdot 01 \\
0.81 \pm 0.36 \\
0 \cdot 22 \rightarrow I \cdot 63\end{array}$ & $\begin{array}{l}0.48 \pm 0.22 \\
0.18 \rightarrow 0.82 \\
0.30 \pm 0.16 \\
0.07 \rightarrow 0.67 \\
0.26 \pm 0.15 \\
0.06 \rightarrow 0.62\end{array}$ \\
\hline$S$ & $\begin{array}{l}\text { (a) } \\
\text { (b) } \\
\text { (c) }\end{array}$ & $\begin{array}{l}0.24 \pm 0.18 \\
0.03 \rightarrow 0.46 \\
0.19 \pm 0.12 \\
0.03 \rightarrow 0.43 \\
0.16 \pm 0.12 \\
0.06 \rightarrow 0.43\end{array}$ & $\begin{array}{l}0.16 \pm 0.13 \\
0.01 \rightarrow 0.34 \\
0.18 \pm 0.13 \\
0.05 \rightarrow 0.41 \\
0.16 \pm 0.12 \\
0.03 \rightarrow 0.47\end{array}$ & $\begin{array}{l}0.69 \pm 0.29 \\
0.34 \rightarrow 1 \cdot 11 \\
0.53 \pm 0.28 \\
0.12 \rightarrow 1.13 \\
0.48 \pm 0.22 \\
0.15 \rightarrow 0.94\end{array}$ \\
\hline $\begin{array}{l}\text { ST } \\
\text { junction }\end{array}$ & $\begin{array}{l}\text { (a) } \\
\text { (b) } \\
\text { (c) }\end{array}$ & $\begin{array}{r}0.02 \pm 0.04 \\
-0.03 \rightarrow 0.05 \\
0.01 \pm 0.03 \\
-0.04 \rightarrow 0.08 \\
0.00 \pm 0.03 \\
-0.06 \rightarrow 0.07\end{array}$ & $\begin{array}{r}0.02 \pm 0.02 \\
-0.01 \rightarrow 0.04 \\
0.02 \pm 0.04 \\
-0.04 \rightarrow 0.11 \\
0.02 \pm 0.04 \\
-0.06 \rightarrow 0.09\end{array}$ & $\begin{array}{r}0.04 \pm 0.03 \\
0.01 \rightarrow 0.08 \\
0.03 \pm 0.03 \\
-0.03 \rightarrow 0.10 \\
0.02 \pm 0.03 \\
-0.04 \rightarrow 0.08\end{array}$ \\
\hline $\mathrm{T}$ & $\begin{array}{l}\text { (a) } \\
\text { (b) } \\
\text { (c) }\end{array}$ & $\begin{array}{l}0.42 \pm 0.20 \\
0.22 \rightarrow 0.75 \\
0.30 \pm 0.13 \\
0.06 \rightarrow 0.50 \\
0.26 \pm 0.10 \\
0.07 \rightarrow 0.44\end{array}$ & $\begin{array}{l}0.26 \pm 0.15 \\
0.11 \rightarrow 0.59 \\
0.26 \pm 0.12 \\
0.11 \rightarrow 0.54 \\
0.25 \pm 0.11 \\
0.09 \rightarrow 0.45\end{array}$ & $\begin{array}{r}0.12 \pm 0.12 \\
0.02 \rightarrow 0.39 \\
0.17 \pm 0.11 \\
-0.05 \rightarrow 0.39 \\
0.16 \pm 0.10 \\
-0.05 \rightarrow 0.34\end{array}$ \\
\hline
\end{tabular}


TABLE 3 Normal ranges of $Q, R, S$ wave durations. Wave onset and terminations determined individually for each lead

\begin{tabular}{|c|c|c|c|c|}
\hline \multirow[t]{2}{*}{ Wave } & & \multicolumn{3}{|l|}{ Lead } \\
\hline & & $X$ & $Y$ & $Z$ \\
\hline Q & $\begin{array}{l}\text { (a) } \\
\text { (b) } \\
\text { (c) }\end{array}$ & $\begin{array}{l}0.018 \pm 0.004 \text { sec. } \\
0.015 \rightarrow 0.024 \\
0.019 \pm 0.006 \\
0.010 \rightarrow 0.027 \\
0.017 \pm 0.004 \\
0.010 \rightarrow 0.023\end{array}$ & $\begin{array}{l}0.020 \pm 0.005 \\
0.013 \rightarrow 0.024 \\
0.019 \pm 0.006 \\
0.010 \rightarrow 0.026 \\
0.019 \pm 0.010 \\
0.009 \rightarrow 0.044\end{array}$ & $\begin{array}{l}- \\
- \\
-\end{array}$ \\
\hline $\mathbf{R}$ & $\begin{array}{l}\text { (a) } \\
\text { (b) } \\
\text { (c) }\end{array}$ & $\begin{array}{l}0.038 \pm 0.009 \\
0.029 \rightarrow 0.052 \\
0.041 \pm 0.010 \\
0.029 \rightarrow 0.068 \\
0.043 \pm 0.013 \\
0.028 \rightarrow 0.084\end{array}$ & $\begin{array}{l}0.044 \pm 0.014 \\
0.034 \rightarrow 0.072 \\
0.048 \pm 0.016 \\
0.016 \rightarrow 0.081 \\
0.050 \pm 0.019 \\
0.028 \rightarrow 0.094\end{array}$ & $\begin{array}{l}0.035 \pm 0.007 \\
0.023 \rightarrow 0.045 \\
0.034 \pm 0.008 \\
0.019 \rightarrow 0.048 \\
0.033 \pm 0.008 \\
0.018 \rightarrow 0.053\end{array}$ \\
\hline$S$ & $\begin{array}{l}\text { (a) } \\
\text { (b) } \\
\text { (c) }\end{array}$ & $\begin{array}{l}0.030 \pm 0.009 \\
0.014 \rightarrow 0.040 \\
0.028 \pm 0.010 \\
0.012 \rightarrow 0.047 \\
0.032 \pm 0.012 \\
0.014 \rightarrow 0.057\end{array}$ & $\begin{array}{l}0.025 \pm 0.009 \\
0.016 \rightarrow 0.040 \\
0.027 \pm 0.008 \\
0.015 \rightarrow 0.040 \\
0.026 \pm 0.009 \\
0.010 \rightarrow 0.045\end{array}$ & $\begin{array}{l}0.038 \pm 0.009 \\
0.028 \rightarrow 0.050 \\
0.042 \pm 0.011 \\
0.020 \rightarrow 0.065 \\
0.043 \pm 0.010 \\
0.024 \rightarrow 0.064\end{array}$ \\
\hline
\end{tabular}

TABLE 5 Normal ranges of magnitude and orientation of maximal spatial vectors

\begin{tabular}{|c|c|c|c|c|c|}
\hline \multirow{2}{*}{\multicolumn{2}{|c|}{$\begin{array}{l}\text { Maximum } \\
\text { spatial } \\
\text { vector }\end{array}$}} & \multicolumn{4}{|l|}{ Plane } \\
\hline & & \multirow{2}{*}{$\begin{array}{l}F \\
\begin{array}{c}43 \pm 49^{\circ} \\
321 \rightarrow 78\end{array}\end{array}$} & \multirow{2}{*}{$\frac{R S}{\substack{54 \pm 55 \\
311 \rightarrow 109}}$} & \multirow{2}{*}{$\begin{array}{l}T \\
20 \pm 42 \\
306 \rightarrow 84\end{array}$} & \multirow{2}{*}{$\begin{array}{l}\text { Amplitude } \\
0.13 \pm 0.04 \\
0.09 \rightarrow 0.20\end{array}$} \\
\hline $\mathbf{P}$ & (a) & & & & \\
\hline & (b) & $\begin{array}{l}54 \pm 43 \\
27 \rightarrow \text { I04 }\end{array}$ & $\begin{array}{l}72 \pm 51 \\
58 \rightarrow 167\end{array}$ & $\begin{array}{c}26 \pm 44 \\
4 \rightarrow 149\end{array}$ & $\begin{array}{l}0.14 \pm 0.05 \\
0.06 \rightarrow 0.26\end{array}$ \\
\hline & (c) & $\begin{array}{c}60 \pm 39 \\
271 \rightarrow 102\end{array}$ & $\begin{array}{l}88 \pm 41 \\
14 \rightarrow 216\end{array}$ & $\begin{array}{c}\mathrm{II} \pm 54 \\
\mathrm{2} \rightarrow \mathrm{I} 2 \mathrm{O}\end{array}$ & $\begin{array}{l}0.16 \pm 0.05 \\
0.07 \rightarrow 0.28\end{array}$ \\
\hline \multirow[t]{4}{*}{ QRS } & (a) & $\begin{array}{l}45 \pm 14 \\
24 \rightarrow 62\end{array}$ & $\begin{array}{l}89 \pm 30 \\
39 \rightarrow 124\end{array}$ & $\begin{array}{l}359 \pm 28 \\
316 \rightarrow 38\end{array}$ & $\begin{array}{l}I .83 \pm 0.54 \\
I \cdot I I \rightarrow 2.5 I\end{array}$ \\
\hline & (b) & $44 \pm 24$ & $102 \pm 21$ & $348 \pm 21$ & $1.69 \pm 0.46$ \\
\hline & & $17 \rightarrow 73$ & $72 \rightarrow 146$ & $305 \rightarrow 23$ & $1 \cdot 05 \rightarrow 2 \cdot 79$ \\
\hline & (c) & $\begin{array}{r}36 \pm 21 \\
0 \rightarrow 64\end{array}$ & $\begin{array}{l}94 \pm 31 \\
30 \rightarrow 150\end{array}$ & $\begin{array}{l}354 \pm 20 \\
318 \rightarrow 19\end{array}$ & $\begin{array}{l}1.42 \pm 0.37 \\
0.89 \rightarrow 2.30\end{array}$ \\
\hline \multirow[t]{4}{*}{$\mathrm{T}$} & (a) & $\begin{array}{l}32 \pm 13 \\
15 \rightarrow 54\end{array}$ & $\begin{array}{l}76 \pm 21 \\
42 \rightarrow 103\end{array}$ & $\begin{array}{r}12 \pm 17 \\
355 \rightarrow 36\end{array}$ & $\begin{array}{l}0.52 \pm 0.23 \\
0.28 \rightarrow 1.03\end{array}$ \\
\hline & (b) & $41 \pm 20$ & $59 \pm 22$ & $29 \pm 21$ & $0.45 \pm 0.15$ \\
\hline & & I7 $\rightarrow 69$ & $27 \rightarrow \mathrm{IOI}$ & $349 \rightarrow 78$ & $0.20 \rightarrow 0.80$ \\
\hline & (c) & $\begin{array}{l}43 \pm 18 \\
16 \rightarrow 70\end{array}$ & $\begin{array}{l}59 \pm 22 \\
25 \rightarrow 92\end{array}$ & $\begin{array}{r}29 \pm 20 \\
358 \rightarrow 65\end{array}$ & $\begin{array}{l}0.40 \pm 0.13 \\
0.18 \rightarrow 0.67\end{array}$ \\
\hline
\end{tabular}

TABLE 4 Normal ranges of $R / Q, R / S, R / T$ ratios

\begin{tabular}{|c|c|c|c|c|}
\hline \multicolumn{2}{|c|}{ Ratio } & \multicolumn{3}{|l|}{ Lead } \\
\hline & & $X$ & $Y$ & $Z$ \\
\hline $\mathrm{R} / \mathrm{Q}$ & $\begin{array}{l}\text { (a) } \\
\text { (b) } \\
\text { (c) }\end{array}$ & $\begin{array}{r}14 \cdot 1 \pm 11 \cdot 3 \\
6 \cdot 7 \rightarrow 26 \cdot 3 \\
17 \cdot 0 \pm 16 \cdot 3 \\
5 \cdot 4 \rightarrow 56 \cdot 4 \\
18 \cdot 0 \pm 17 \cdot 2 \\
5 \cdot 2 \rightarrow 59 \cdot 1\end{array}$ & $\begin{array}{r}13 \cdot 0 \pm 6 \cdot 3 \\
7 \cdot 1 \rightarrow 21 \cdot 2 \\
14 \cdot 5 \pm 16 \cdot 1 \\
3 \cdot 8 \rightarrow 49 \cdot 8 \\
13 \cdot 0 \pm 13 \cdot 0 \\
3 \cdot 0 \rightarrow 40 \cdot 3\end{array}$ & $\begin{array}{l}- \\
- \\
-\end{array}$ \\
\hline$R / S$ & $\begin{array}{l}\text { (a) } \\
\text { (b) } \\
\text { (c) }\end{array}$ & $\begin{array}{l}- \\
- \\
-\end{array}$ & $\begin{array}{l}- \\
- \\
-\end{array}$ & $\begin{array}{r}\mathrm{I} \cdot 0 \pm \mathrm{I} \cdot 3 \\
0 \cdot 3 \rightarrow 2 \cdot 7 \\
0.69 \pm \mathrm{I} \cdot 3 \\
0 \cdot \mathrm{I} \rightarrow 2 \cdot 2 \\
0.68 \pm 0.9 \\
0.1 \rightarrow \mathrm{I} \cdot 4\end{array}$ \\
\hline $\mathbf{R} / \mathbf{T}$ & $\begin{array}{l}\text { (a) } \\
\text { (b) } \\
\text { (c) }\end{array}$ & $\begin{array}{l}3 \cdot 3 \pm 1 \cdot 04 \\
1 \cdot 7 \rightarrow 4 \cdot 4 \\
3 \cdot 8 \pm 4 \cdot 1 \\
0 \cdot 1 \rightarrow 12 \cdot 7 \\
4 \cdot 6 \pm 3 \cdot 3 \\
0 \cdot 3 \rightarrow 10 \cdot 2\end{array}$ & $\begin{array}{l}5.6 \pm 3.1 \\
2.0 \rightarrow 10.7 \\
4.6 \pm 2.9 \\
0.01 \rightarrow 12.9 \\
3.4 \pm 2.3 \\
0.3 \rightarrow 8.3\end{array}$ & $\begin{array}{c}5.6 \pm 5.3 \\
0.5 \rightarrow 16.9 \\
2.4 \pm 7.0 \\
-3.5 \rightarrow 8.0 \\
2.0 \pm 2.8 \\
0.7 \rightarrow 6.4\end{array}$ \\
\hline
\end{tabular}

TABLE 6 Normal ranges of amplitude of maximum vector in each plane. Each column is derived independently of others

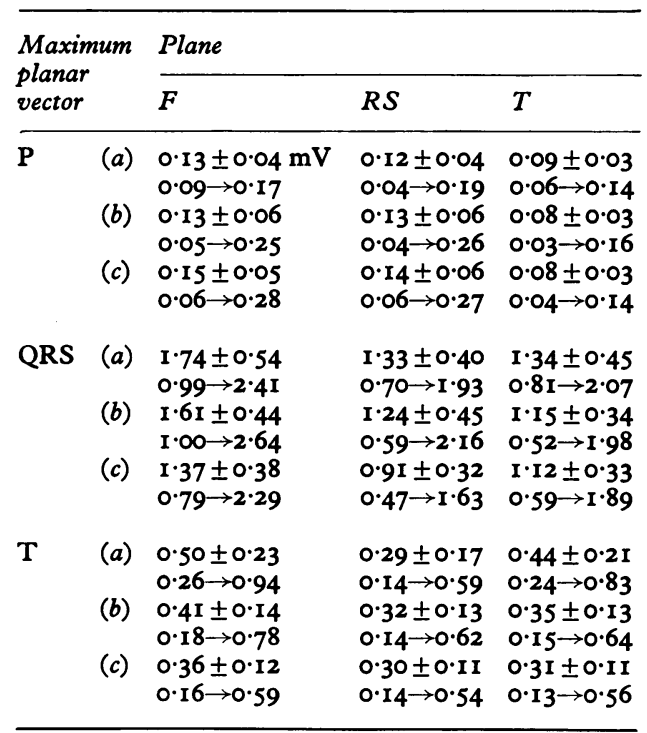

Maximum $P, Q R S$, and $T$ vector magnitudes and orientations were calculated together with the vectors at $0.01,0.02$, and $0.03 \mathrm{sec}$. after QRS onset and $-0.01,-0.02$, and $-0.03 \mathrm{sec}$. before QRS termination (Fig. 2). The projection of the maximum vector on to each plane and the maximum vector in each plane (not necessarily the same) were also calculated. Only the early QRS vectors were found to be of diagnostic significance in subsequent clinical studies.

The $R / S, R / Q$, and $R / T$ ratios were calculated for each lead. Time integrals were also obtained, and though these were not extensively utilized in the interpretation programme their values are presented for completeness.

\section{Results}

The results of the study are presented in Tables 2-13. Means and standard deviations together with 96 per cent ranges are given for each age-group. 
TABLE 7 Normal ranges of orientation of maximum vectors in each plane. Each column is derived independently of others

\begin{tabular}{|c|c|c|c|c|}
\hline \multirow{2}{*}{\multicolumn{2}{|c|}{$\begin{array}{l}\text { Maximum } \\
\text { planar } \\
\text { vector }\end{array}$}} & \multicolumn{3}{|l|}{ Plane } \\
\hline & & \multirow{2}{*}{$\begin{array}{l}F \\
\begin{array}{r}40 \pm 45^{\circ} \\
340 \rightarrow 70\end{array}\end{array}$} & \multirow{2}{*}{$\begin{array}{l}R S \\
\stackrel{27 \pm 64}{300 \stackrel{108}{\rightarrow}}\end{array}$} & \multirow{2}{*}{$\frac{T}{\substack{21 \pm 50 \\
314 \rightarrow 10 I}}$} \\
\hline $\mathbf{P}$ & (a) & & & \\
\hline & (b) & $\begin{array}{l}49 \pm 42 \\
27 \rightarrow 99\end{array}$ & $\begin{array}{l}64 \pm 45 \\
41 \rightarrow 108\end{array}$ & $\begin{aligned} 25 & \pm 53 \\
2 & \rightarrow 165\end{aligned}$ \\
\hline & (c) & $\begin{array}{r}62 \pm 36 \\
352 \rightarrow 99\end{array}$ & $\begin{array}{c}86 \pm 4 I \\
I \rightarrow 23 I\end{array}$ & $\begin{array}{l}3 \pm 54 \\
2 \rightarrow 103\end{array}$ \\
\hline \multicolumn{2}{|c|}{ QRS (a) } & $\begin{array}{l}42 \pm I 4 \\
27 \rightarrow 62\end{array}$ & $\begin{array}{c}102 \pm 30 \\
52 \rightarrow 155\end{array}$ & $\begin{array}{l}335 \pm 56 \\
235 \rightarrow 40\end{array}$ \\
\hline & (b) & $\begin{array}{l}45 \pm 14 \\
17 \rightarrow 74\end{array}$ & $\begin{array}{c}110 \pm 33 \\
64 \rightarrow 209\end{array}$ & $\begin{array}{l}349 \pm 26 \\
272 \rightarrow 30\end{array}$ \\
\hline & $(c)$ & $\begin{array}{r}35 \pm 19 \\
0 \rightarrow 63\end{array}$ & $\begin{array}{c}109 \pm 42 \\
49 \rightarrow 210\end{array}$ & $\begin{array}{l}355 \pm 24 \\
278 \rightarrow 23\end{array}$ \\
\hline \multirow[t]{4}{*}{$\mathbf{T}$} & (a) & $\begin{array}{l}32 \pm 13 \\
15 \rightarrow 54\end{array}$ & $\begin{array}{l}76 \pm 21 \\
46 \rightarrow 106\end{array}$ & $\begin{aligned} \underset{355}{12 \cdot 5} \rightarrow \mathbf{3 6} & \pm 17 \\
& \end{aligned}$ \\
\hline & (b) & $41 \pm 20$ & $58 \pm 23$ & $30 \pm 21$ \\
\hline & & I7 $\rightarrow 70$ & $27 \rightarrow 102$ & $352 \rightarrow 78$ \\
\hline & (c) & $\begin{array}{l}42 \pm 18 \\
15 \rightarrow 68\end{array}$ & $\begin{array}{l}59 \pm 22 \\
25 \rightarrow 92\end{array}$ & $\begin{array}{r}29 \pm 23 \\
357 \rightarrow 71\end{array}$ \\
\hline
\end{tabular}

While it is accepted that the onset of the QRS complex must occur simultaneously in all leads, there is not always simultaneous movement from the baseline in each lead. It should be noted that the $Q_{Y}$ wave duration, for example, is based on a measurement from the initial deviation from the baseline in lead $Y$, which is not necessarily at the same instant as the first deviation of any one of the group of 3 leads. This method was adopted since it may be that 3 lead electrocardiograms are interpreted without computer assistance in which case it is easier to measure the $Q$ wave duration, etc., in each lead separately. In practice diagnostic criteria used in subsequent work incorporated the $0.03 \mathrm{sec}$. vector, which is referred to the simultaneous (approximate) onset as determined from the QRS spatial velocity.

Table I4 shows the values of the lead strengths for the modified and unmodified axial lead systems. From these data it is possible to calculate ranges for the unmodified axial system as shown in the Appendix.

\section{Discussion}

For two reasons no further attempt was made to classify the electrocardiogram measurements by sex. Firstly this is rarely done in - routine practice, and secondly such a subdivision would have reduced the numbers in ' each group. The total of 18 patients in the under-15 age-group is admittedly low but sufficient to emphasize the importance of
TABLE 8 Normal ranges of magnitude and orientation of the 8 time-normalized $Q R S$ vectors

\begin{tabular}{|c|c|c|c|c|c|}
\hline \multirow[t]{2}{*}{ Vector } & & \multicolumn{4}{|l|}{ Plane } \\
\hline & & $F$ & $R S$ & $T$ & Amplitude in $m V$ \\
\hline \multirow[t]{5}{*}{$\begin{array}{l}\text { I/8 } \\
\text { QRS }\end{array}$} & (a) & $\begin{array}{l}218 \pm 56^{\circ} \\
126 \rightarrow 285\end{array}$ & $\begin{array}{l}335 \pm 41 \\
294 \rightarrow 38\end{array}$ & $\begin{array}{c}120 \pm 45 \\
70 \rightarrow 158\end{array}$ & $\begin{array}{l}0.17 \pm 0.10 \\
0.03 \rightarrow 0.27\end{array}$ \\
\hline & (b) & $211 \pm 72$ & $336 \pm 45$ & $106 \pm 42$ & $0.13 \pm 0.07$ \\
\hline & & $52 \rightarrow 327$ & $271 \rightarrow 73$ & $5 \rightarrow 169$ & $0.02 \rightarrow 0.30$ \\
\hline & (c) & $205 \pm 76$ & $346 \pm 59$ & $117 \pm 52$ & $0.10 \pm 0.05$ \\
\hline & & $64 \rightarrow \mathrm{II}$ & $204 \rightarrow \mathrm{II} 4$ & $16 \rightarrow 255$ & $0.02 \rightarrow 0.21$ \\
\hline \multirow{4}{*}{$\begin{array}{l}2 / 8 \\
\text { QRS }\end{array}$} & (a) & $64 \pm 80$ & $16 \pm 26$ & $69 \pm 35$ & $0.47 \pm 0.20$ \\
\hline & & $349 \rightarrow 191$ & $350 \rightarrow 65$ & $33 \rightarrow 122$ & $0.30 \rightarrow 0.74$ \\
\hline & (b) & $\begin{array}{l}26 \pm 85 \\
16 \rightarrow 182\end{array}$ & $\begin{array}{r}17 \pm 36 \\
8 \rightarrow 89\end{array}$ & $\begin{array}{c}72 \pm 39 \\
I \rightarrow 154\end{array}$ & $\begin{array}{l}0.35 \pm 0.15 \\
0.11 \rightarrow 0.69\end{array}$ \\
\hline & $(c)$ & $\begin{array}{l}54 \pm 66 \\
15 \rightarrow 194\end{array}$ & $\begin{array}{r}22 \pm 37 \\
3 \rightarrow 82\end{array}$ & $\begin{array}{c}62 \pm 39 \\
3 \rightarrow 141\end{array}$ & $\begin{array}{l}0.32 \pm 0.18 \\
0.08 \rightarrow 0.71\end{array}$ \\
\hline \multirow[t]{4}{*}{$\begin{array}{l}3 / 8 \\
\text { QRS }\end{array}$} & (a) & $\begin{array}{l}43 \pm 12 \\
3 I \rightarrow 60\end{array}$ & $\begin{array}{l}77 \pm 30 \\
31 \rightarrow 118\end{array}$ & $\begin{array}{r}10 \pm 31 \\
338 \rightarrow 46\end{array}$ & $\begin{array}{l}I \cdot 35 \pm 0.49 \\
0.85 \rightarrow 1.91\end{array}$ \\
\hline & (b) & $39 \pm 26$ & $78 \pm 29$ & $10 \pm 27$ & $1.08 \pm 0.45$ \\
\hline & (c) & $\begin{array}{r}333 \rightarrow 71 \\
33 \pm 24\end{array}$ & $\begin{array}{c}345 \rightarrow 128 \\
78 \pm 34\end{array}$ & $7 \pm 24$ & $1.01 \pm 0.33$ \\
\hline & & $0 \rightarrow 58$ & $22 \rightarrow 131$ & $I \rightarrow 47$ & $0.44 \rightarrow \mathrm{I} .6 \mathrm{I}$ \\
\hline \multirow[t]{5}{*}{$\begin{array}{l}4 / 8 \\
\text { QRS }\end{array}$} & (a) & $\begin{array}{l}68 \pm 40 \\
32 \rightarrow 150\end{array}$ & $\begin{array}{c}118 \pm 32 \\
69 \rightarrow 161\end{array}$ & $\begin{array}{l}315 \pm 53 \\
234 \rightarrow 28\end{array}$ & $\begin{array}{l}1.43 \pm 0.49 \\
0.85 \rightarrow 2.23\end{array}$ \\
\hline & (b) & $55 \pm 38$ & $117 \pm 27$ & $329 \pm 33$ & $1 \cdot 34 \pm 0.49$ \\
\hline & & $I I \rightarrow 192$ & $75 \rightarrow 185$ & $256 \rightarrow 16$ & $0.43 \rightarrow 2.27$ \\
\hline & (c) & $37 \pm 42$ & $126 \pm 41$ & $329 \pm 37$ & $1 \cdot 08 \pm 0.46$ \\
\hline & & $9 \rightarrow \operatorname{II2}$ & $73 \rightarrow 215$ & $238 \rightarrow 10$ & $0.35 \rightarrow 2 \cdot \mathrm{II}$ \\
\hline \multirow{6}{*}{$\begin{array}{l}5 / 8 \\
\text { QRS }\end{array}$} & (a) & $132 \pm 94^{\circ}$ & $175 \pm 27$ & $261 \pm 35$ & $0.59 \pm 0.24$ \\
\hline & & $357 \rightarrow 264$ & $137 \rightarrow 206$ & $218 \rightarrow 311$ & $0.32 \rightarrow 0.75$ \\
\hline & (b) & $86 \pm 88$ & $163 \pm 39$ & $273 \pm 40$ & $0.65 \pm 0.34$ \\
\hline & & $39 \rightarrow 234$ & $84 \rightarrow 238$ & $208 \rightarrow 5$ & $0.15 \rightarrow 1.36$ \\
\hline & (c) & $264 \pm$ II 4 & $169 \pm 4 I$ & $278 \pm 39$ & $0.54 \pm 0.26$ \\
\hline & & $8 I \rightarrow 64$ & $101 \rightarrow 231$ & $210 \rightarrow 350$ & $0.2 \mathrm{I} \rightarrow \mathrm{I} \cdot 18$ \\
\hline \multirow{6}{*}{$\begin{array}{l}\text { 6/8 } \\
\text { QRS }\end{array}$} & (a) & $269 \pm 72$ & $219 \pm 44$ & $252 \pm 50$ & $0.22 \pm 0.13$ \\
\hline & & $156 \rightarrow 52$ & $146 \rightarrow 277$ & $186 \rightarrow 334$ & $0.06 \rightarrow 0.33$ \\
\hline & (b) & $195 \pm 82$ & $189 \pm 57$ & $240 \pm 58$ & $0.23 \pm 0.12$ \\
\hline & & $55 \rightarrow 3$ & $85 \rightarrow 290$ & $9 I \rightarrow 340$ & $0.04 \pm 0.51$ \\
\hline & (c) & $35^{8} \pm 110$ & $179 \pm 55$ & $258 \pm 51$ & $0.21 \pm 0.12$ \\
\hline & & $187 \rightarrow \mathrm{I} 65$ & $95 \rightarrow 280$ & $120 \rightarrow 10$ & $0.96 \rightarrow 0.49$ \\
\hline \multirow{6}{*}{$\begin{array}{l}7 / 8 \\
\text { QRS }\end{array}$} & (a) & $20 \pm 74$ & $32 \pm 64$ & $6 I \pm 64$ & $0.07 \pm 0.04$ \\
\hline & & $238 \rightarrow 115$ & $301 \rightarrow 116$ & $344 \rightarrow$ I 57 & $0.04 \rightarrow 0.13$ \\
\hline & (b) & $71 \pm 95$ & $54 \pm 87$ & $44 \pm 91$ & $0.07 \pm 0.04$ \\
\hline & & $9 \rightarrow 260$ & $9 \rightarrow 203$ & $13 \rightarrow 208$ & $0.01 \rightarrow 0.15$ \\
\hline & (c) & $55 \pm 99$ & $102 \pm 94$ & $357 \pm 106$ & $0.07 \pm 0.04$ \\
\hline & & $16 \rightarrow 222$ & $19 \rightarrow 270$ & $185 \rightarrow 166$ & $0.01 \rightarrow 0.15$ \\
\hline \multirow{6}{*}{$\begin{array}{l}8 / 8 \\
\text { QRS }\end{array}$} & (a) & $50 \pm 76$ & $6 \pm 37$ & $78 \pm 53$ & $0.08 \pm 0.04$ \\
\hline & & $330 \rightarrow 200$ & $316 \rightarrow 4 I$ & $25 \rightarrow 159$ & $0.03 \rightarrow 0.15$ \\
\hline & (b) & $67 \pm 77$ & $27 \pm 50$ & $67 \pm 50$ & $0.07 \pm 0.04$ \\
\hline & & $19 \rightarrow 234$ & $2 \rightarrow 117$ & $16 \rightarrow 158$ & $0.02 \rightarrow 0.17$ \\
\hline & (c) & $\begin{array}{l}60 \pm 84 \\
14 \rightarrow 208\end{array}$ & $\begin{array}{l}30 \pm 60 \\
1 I \rightarrow 166\end{array}$ & $80 \pm 64$ & $\begin{array}{l}0.06 \pm 0.02 \\
0.02 \rightarrow 0.12\end{array}$ \\
\hline & & $14 \rightarrow 208$ & I I $\rightarrow$ I 66 & $24 \rightarrow 194$ & $0.02 \rightarrow 0.12$ \\
\hline
\end{tabular}


TABLE 9 Normal ranges of component amplitudes of time-normalized $Q R S$ vectors

classification by age. Gamboa and White (I968) have already published unmodified axial lead system data for children. parameters is presented, further study showed some to be of more use than others. In particular, the projection of the maximum spatial vector on to the transverse plane had a narrower range of values than the maximum vector in the plane and so proved to be the better diagnostic parameter (Fig. 3). This is of considerable significance in the visual interpretation of vectorcardiograms where a false positive diagnosis of left ventricular transverse plane vector orientations alone if a careful scrutiny of the vectorcardiogram is not made.

The concept of time normalization ultimST-T abnormalities. Fig. 4 shows how early or late ST-T abnormalities could be overlooked if only the maximum $T$ wave amplitude were considered. On the other hand, the 0.03 sec., etc., QRS vectors proved more useful than the time-normalized QRS vectors.

There is no doubt that the greater number of projections of the cardiac electrical activity in the 12 lead electrocardiogram may make abnormalities more apparent than in the 3 lead, but the use of computer techniques to derive the parameters presented above leads to equally accurate clinical interpretations in each case (Macfarlane, Lorimer, and Lawrie, I97I). This is of considerable importance in gram interpretation, since there is an obvious saving in time for analysis compared to the 12 lead electrocardiogram.
While a full selection of electrocardiogram hypertrophy could be made on the basis of ately proved of great use in the diagnosis of the application of computers to electrocardio-

FIG. 4 (a) Early ST-T abnormalities at approximately the 2/8 amplitude. (b) Late $S T-T$ abnormalities at approximately the 6/8 amplitude. Neither abnormality would be detected by a check on the maximum $T$ wave amplitude denoted by the arrow. This is not the case with the use of time-normalized data.

\begin{tabular}{|c|c|c|c|c|}
\hline \multirow[t]{2}{*}{ Vector } & & \multicolumn{3}{|l|}{ Lead } \\
\hline & & $X$ & $Y$ & $Z$ \\
\hline $\begin{array}{l}\text { I/8 } \\
\text { QRS }\end{array}$ & $\begin{array}{l}\text { (a) } \\
(b) \\
(c)\end{array}$ & $\begin{array}{l}-0.07 \pm 0.07 \mathrm{mV} \\
-0.17 \rightarrow 0.03 \\
-0.03 \pm 0.06 \\
-0.18 \rightarrow 0.11 \\
-0.03 \pm 0.05 \\
-0.14 \rightarrow 0.06\end{array}$ & $\begin{array}{l}-0.04 \pm 0.09 \\
-0.14 \rightarrow 0.05 \\
-0.04 \pm 0.07 \\
-0.17 \rightarrow 0.12 \\
-0.01 \pm 0.06 \\
-0.12 \rightarrow 0.12\end{array}$ & $\begin{array}{r}0.11 \pm 0.08 \\
0.02 \rightarrow 0.21 \\
0.08 \pm 0.06 \\
0.0 \rightarrow 0.23 \\
0.06 \pm 0.05 \\
-0.02 \rightarrow 0.16\end{array}$ \\
\hline $\begin{array}{l}2 / 8 \\
\text { QRS }\end{array}$ & $\begin{array}{l}\text { (a) } \\
(b) \\
(c)\end{array}$ & $\begin{array}{r}0.17 \pm 0.27 \\
-0.19 \rightarrow 0.50 \\
0.10 \pm 0.19 \\
-0.17 \rightarrow 0.55 \\
0.13 \pm 0.20 \\
-0.17 \rightarrow 0.60\end{array}$ & $\begin{array}{r}0.08 \pm 0.14 \\
-0.06 \rightarrow 0.24 \\
0.08 \pm 0.16 \\
-0.25 \rightarrow 0.39 \\
0.11 \pm 0.13 \\
-0.12 \rightarrow 0.36\end{array}$ & $\begin{array}{l}0.34 \pm 0.15 \\
0.16 \rightarrow 0.53 \\
0.23 \pm 0.13 \\
0.0 \rightarrow 0.52 \\
0.19 \pm 0.11 \\
0.01 \rightarrow 0.44\end{array}$ \\
\hline $\begin{array}{l}\text { 3/8 } \\
\text { QRS }\end{array}$ & $\begin{array}{l}\text { (a) } \\
(b) \\
(c)\end{array}$ & $\begin{array}{l}0.92 \pm 0.46 \\
0.32 \rightarrow 1 \cdot 60 \\
0.74 \pm 0.37 \\
0.07 \rightarrow 1 \cdot 62 \\
0.78 \pm 0.30 \\
0.19 \rightarrow 1 \cdot 45\end{array}$ & $\begin{array}{r}0.83 \pm 0.35 \\
0.42 \rightarrow 1.44 \\
0.67 \pm 0.38 \\
-0.08 \rightarrow 1.47 \\
0.53 \pm 0.29 \\
0.0 \rightarrow 1.03\end{array}$ & $\begin{array}{r}0.15 \pm 0.42 \\
-0.39 \rightarrow 0.75 \\
0.07 \pm 0.28 \\
-0.56 \rightarrow 0.54 \\
0.09 \pm 0.24 \\
-0.45 \rightarrow 0.51\end{array}$ \\
\hline $\begin{array}{l}4 / 8 \\
\text { QRS }\end{array}$ & $\begin{array}{l}\text { (a) } \\
(b) \\
(c)\end{array}$ & $\begin{array}{r}0.61 \pm 0.74 \\
-0.47 \rightarrow 1.73 \\
0.76 \pm 0.46 \\
-0.15 \rightarrow 1.61 \\
0.69 \pm 0.49 \\
0.18 \rightarrow 1.63\end{array}$ & $\begin{array}{r}0.86 \pm 0.42 \\
0.27 \rightarrow 1.56 \\
0.87 \pm 0.49 \\
-0.07 \rightarrow \mathbf{1} .89 \\
0.55 \pm 0.47 \\
-0.33 \rightarrow 1.56\end{array}$ & $\begin{array}{l}-0.46 \pm 0.53 \\
-1.12 \rightarrow 0.35 \\
-0.35 \pm 0.36 \\
-1.09 \rightarrow 0.30 \\
-0.28 \pm 0.28 \\
-0.78 \rightarrow 0.18\end{array}$ \\
\hline $\begin{array}{l}5 / 8 \\
\text { QRS }\end{array}$ & $\begin{array}{l}(a) \\
(b) \\
(c)\end{array}$ & $\begin{aligned} &-0.04 \pm 0.28 \mathrm{mV} \\
&-0.34 \rightarrow 0.42 \\
& 0.07 \pm 0.34 \\
&-0.39 \rightarrow 0.96 \rightarrow 0.07 \pm 0.30 \\
&-0.33 \rightarrow 0.72\end{aligned}$ & $\begin{array}{r}0.06 \pm 0.30 \\
-0.22 \rightarrow 0.29 \\
0.21 \pm 0.38 \\
-0.34 \rightarrow 1.26 \\
0.09 \pm 0.28 \\
-0.36 \rightarrow 0.67\end{array}$ & $\begin{array}{l}-0.45 \pm 0.19 \\
-0.68 \rightarrow-0.20 \\
-0.41 \pm 0.26 \\
-0.91 \rightarrow 0.09 \\
-0.37 \pm 0.20 \\
-0.80 \rightarrow-0.02\end{array}$ \\
\hline $\begin{array}{l}6 / 8 \\
\text { QRS }\end{array}$ & $\begin{array}{l}\text { (a) } \\
\text { (b) } \\
\text { (c) }\end{array}$ & $\begin{array}{l}-0.03 \pm 0.10 \\
-0.13 \rightarrow 0.15 \\
-0.05 \pm 0.10 \\
-0.26 \rightarrow 0.11 \\
-0.03 \pm 0.09 \\
-0.22 \rightarrow 0.17\end{array}$ & $\begin{array}{r}-0.08 \pm 0.11 \\
-0.26 \rightarrow 0.06 \\
-0.03 \pm 0.14 \\
-0.27 \rightarrow 0.23 \\
0.0 \pm 0.14 \\
-0.27 \rightarrow 0.28\end{array}$ & $\begin{array}{l}-0.13 \pm 0.15 \\
-0.27 \rightarrow 0.02 \\
-0.14 \pm 0.13 \\
-0.44 \rightarrow 0.09 \\
-0.13 \pm 0.11 \\
-0.40 \rightarrow 0.03\end{array}$ \\
\hline $\begin{array}{l}7 / 8 \\
\text { QRS }\end{array}$ & $\begin{array}{l}\text { (a) } \\
(b) \\
(c)\end{array}$ & $\begin{array}{r}0.03 \pm 0.04 \\
-0.03 \rightarrow 0.08 \\
0.01 \pm 0.04 \\
-0.06 \rightarrow 0.08 \\
0.0 \pm 0.03 \\
-0.06 \rightarrow 0.07\end{array}$ & $\begin{array}{r}0.02 \pm 0.04 \\
-0.03 \rightarrow 0.09 \\
0.01 \pm 0.05 \\
-0.07 \rightarrow 0.10 \\
0.02 \pm 0.05 \\
-0.07 \rightarrow 0.11\end{array}$ & $\begin{array}{r}0.03 \pm 0.04 \\
-0.02 \rightarrow 0.06 \\
0.01 \pm 0.04 \\
-0.07 \rightarrow 0.10 \\
0.0 \pm 0.04 \\
-0.09 \rightarrow 0.08\end{array}$ \\
\hline $\begin{array}{l}8 / 8 \\
\text { QRS }\end{array}$ & $\begin{array}{l}\text { (a) } \\
(b) \\
(c)\end{array}$ & $\begin{array}{r}0.02 \pm 0.04 \\
-0.01 \rightarrow 0.08 \\
0.02 \pm 0.04 \\
-0.04 \rightarrow 0.10 \\
0.01 \pm 0.03 \\
-0.06 \rightarrow 0.07\end{array}$ & $\begin{array}{r}0.01 \pm 0.02 \\
-0.03 \rightarrow 0.06 \\
0.02 \pm 0.04 \\
-0.08 \rightarrow 0.11 \\
0.02 \pm 0.04 \\
-0.06 \rightarrow 0.09\end{array}$ & $\begin{array}{r}0.05 \pm 0.03 \\
0.02 \rightarrow 0.08 \\
0.04 \pm 0.04 \\
-0.03 \rightarrow 0.13 \\
0.03 \pm 0.03 \\
-0.03 \rightarrow 0.11\end{array}$ \\
\hline
\end{tabular}

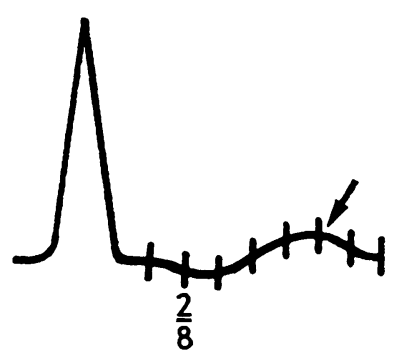

(a) 


\section{References}

American Heart Association (1967). Report of Committee on Electrocardiography. Recommendations for standardization of leads and of specifications for instruments in electrocardiography and vectorcardiography. Circulation, 35, 583.

Draper, H. W., Peffer, C. J., Stallmann, F. W., Littmann, D., and Pipberger, H. V. (1964). The corrected orthogonal electrocardiogram and vectorcardiogram in 510 normal men (Frank lead system). Circulation, 30, 853.

Gamboa, R., and White, N. (1968). The corrected orthogonal electrocardiogram in normal children. McFee and Parungao lead system. American Heart Fournal, 75, 449.

Macfarlane, P. W. (1969). A modified axial lead system for orthogonal lead electrocardiography. Cardiovascular Research, 3, 510.

(I97I). ECG waveform identification by digital computer. Cardiovascular Research, 5, I4I.

- Lorimer, A. R. L., and Lawrie, T. D. V. (1971). 3 and 12 lead electrocardiogram interpretation by computer; a comparison on 1093 patients. British Heart fournal; 33, 266.

McFee, R., and Parungao, A. (196r). An orthogonal lead system for clinical electrocardiography. American Heart fournal, 62, 93.

Simonson, E. (196I). Differentiation between Normal and Abnormal in Electrocardiography. C. V. Mosby, St. Louis.

Stallmann, F. W., and Pipberger, H. V. (196r). Automatic recognition of electrocardiographic waves by digital computer. Circulation Research, 9, II 38 .

\section{Appendix}

From the values of lead strengths as shown in Table 14 it is possible to convert some of the data obtained from the modified system for use with the unmodified system. This applies to wave amplitudes and vector orientations.

To convert lead $Z$ amplitudes each value given in the Tables should be multiplied by $2 \cdot 24 / \mathrm{r} \cdot 48$. Similarly, for lead $X$ the scaling factor is $\mathrm{I} \cdot 6 \mathrm{I} / \mathrm{I} \cdot 48$. Lead $Y$ values do not require any correction.

For the conversion of angular data, let $\theta$ $(0<\theta<90)$ be the angle measured by the modified system, and $\theta^{\prime}$ the angle measured by the unmodified system. Then the following conversion equations are obtained:

$$
\begin{aligned}
& \theta^{\prime} \text { frontal }=\tan ^{-1}\left(\frac{\mathrm{I} \cdot 48}{\mathrm{I} \cdot 6 \mathrm{I}} \tan \theta\right) 0<\theta<90 \\
& \theta^{\prime} \mathrm{R} \text { sagittal }=\tan ^{-1}\left(\frac{\mathrm{I} \cdot 48}{\mathrm{2 \cdot 24}} \tan \theta\right) \\
& \theta^{\prime} \text { transverse }=\tan ^{-1}\left(\frac{2 \cdot 24}{\mathrm{I} \cdot 6 \mathrm{I}} \tan \theta\right)
\end{aligned}
$$

As an example the range of the projection of the maximum QRS spatial vector (Table 5) on to the transverse plane, which is $318^{\circ} \rightarrow 19^{\circ}$ for the modified axial lead system, would be $309^{\circ} \rightarrow 25^{\circ}$ for the original axial lead system (patients over 29 years). This illustrates that angles outside the range $\circ \rightarrow 90^{\circ}$ should be expressed as $0^{\circ} \pm \theta$ or $180^{\circ} \pm \theta$, where $0<\theta<90$, and the conversion made from $\theta$ to $\theta^{\prime}$ so that the new value becomes $0^{\circ} \pm \theta^{\prime}$ or $180^{\circ} \pm \theta^{\prime}$ respectively.

It is not possible to calculate the range of vector magnitudes for the original axial system unless the corresponding individual component amplitudes of leads $\mathrm{X}, \mathrm{Y}$, and $\mathrm{Z}$ are known.
TABLE IO Normal ranges of component amplitudes of time-normalized $S T-T$ vectors

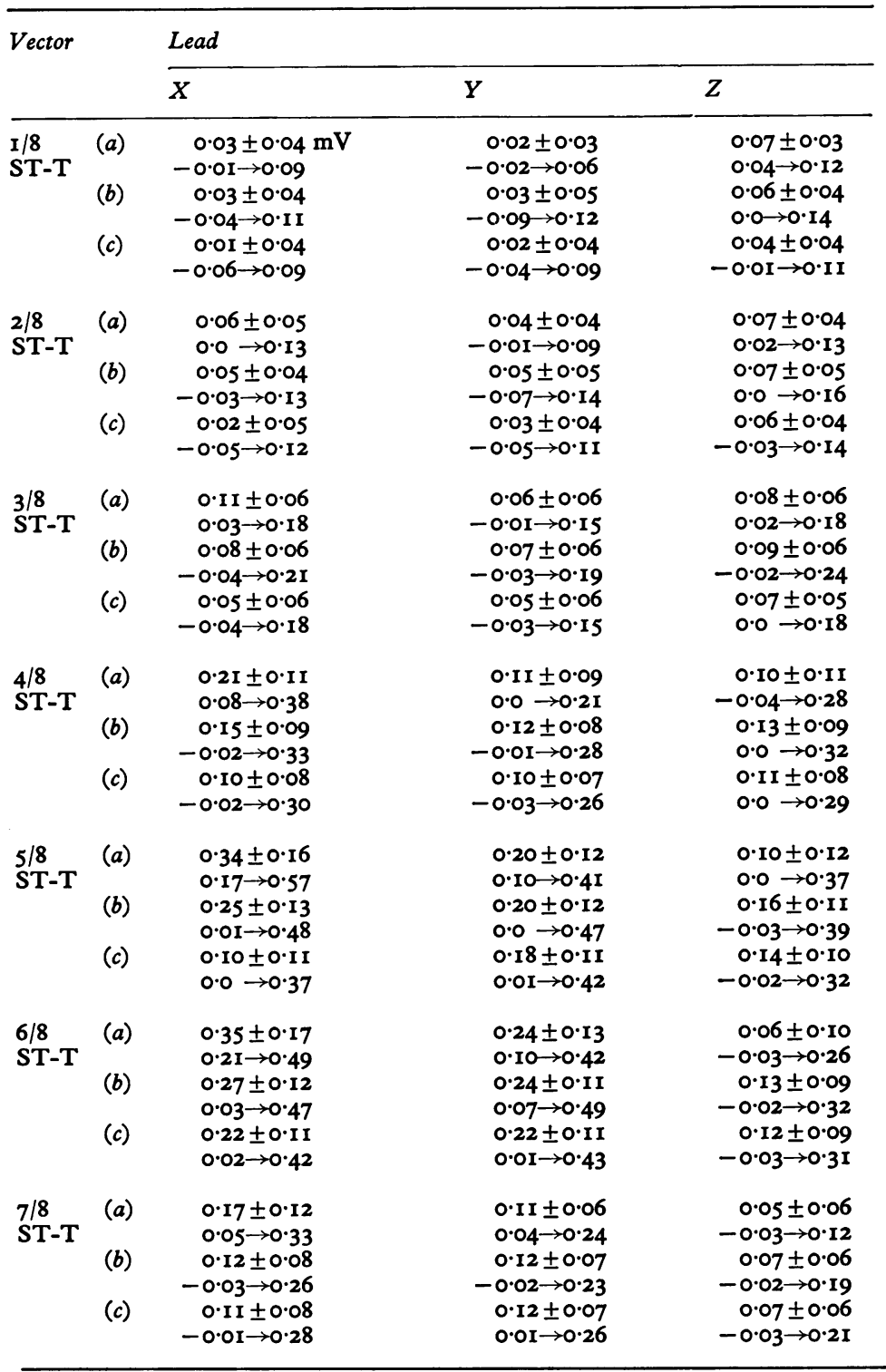

Tables II, I2, I3, and I4 are overleaf. 
TABLE II Normal ranges of $0.01,0.02,0.03$ sec. vectors after $Q R S$ onset and before $Q R S$ termination

\begin{tabular}{|c|c|c|c|c|c|}
\hline \multirow[t]{2}{*}{ Vector } & & \multicolumn{4}{|l|}{ Plane } \\
\hline & & $F$ & $R S$ & $T$ & Amplitude \\
\hline $\begin{array}{l}\text { O.or sec. } \\
\text { after QRS } \\
\text { onset }\end{array}$ & $\begin{array}{l}\text { (a) } \\
\text { (b) } \\
\text { (c) }\end{array}$ & $\begin{array}{c}189 \pm 84^{\circ} \\
65 \rightarrow 298 \\
238 \pm 83 \\
77 \rightarrow 54 \\
210 \pm 80 \\
57 \rightarrow 9\end{array}$ & $\begin{array}{l}352 \pm 56 \\
293 \rightarrow 94 \\
339 \pm 54 \\
263 \rightarrow 95 \\
343 \pm 66 \\
214 \rightarrow 118\end{array}$ & $\begin{array}{c}109 \pm 54 \\
30 \rightarrow 160 \\
103 \pm 57 \\
351 \rightarrow 249 \\
115 \pm 64 \\
44 \rightarrow 253\end{array}$ & $\begin{array}{l}0.08 \pm 0.05 \\
0.02 \rightarrow 0.16 \\
0.07 \pm 0.03 \\
0.01 \rightarrow 0.14 \\
0.06 \pm 0.03 \\
0.01 \rightarrow 0.13\end{array}$ \\
\hline $\begin{array}{l}0.02 \text { sec. } \\
\text { after } Q R S \\
\text { onset }\end{array}$ & $\begin{array}{l}\text { (a) } \\
\text { (b) } \\
(c)\end{array}$ & $\begin{aligned} 205 & \pm 78 \\
92 & \rightarrow 340 \\
234 & \pm 80 \\
76 & \rightarrow 37 \\
125 & \pm 98 \\
23 & \rightarrow 280\end{aligned}$ & $\begin{array}{l}353 \pm 30 \\
315 \rightarrow 44 \\
349 \pm 36 \\
286 \rightarrow 66 \\
357 \pm 48 \\
260 \rightarrow 88\end{array}$ & $\begin{aligned} 102 & \pm 24 \\
60 & \rightarrow 121 \\
101 & \pm 35 \\
25 & \rightarrow 171 \\
100 & \pm 45 \\
2 & \rightarrow 180\end{aligned}$ & $\begin{array}{l}0.26 \pm 0.10 \\
0.13 \rightarrow 0.41 \\
0.22 \pm 0.11 \\
0.03 \rightarrow 0.47 \\
0.17 \pm 0.09 \\
0.04 \rightarrow 0.34\end{array}$ \\
\hline $\begin{array}{l}0.03 \text { sec. } \\
\text { after QRS } \\
\text { onset }\end{array}$ & $\begin{array}{l}\text { (a) } \\
(b) \\
(c)\end{array}$ & $\begin{array}{c}45 \pm 63 \\
6 \rightarrow 153 \\
34 \pm 61 \\
18 \rightarrow 149 \\
31 \pm 53 \\
15 \rightarrow 139\end{array}$ & $\begin{array}{c}31 \pm 32 \\
1 \rightarrow 84 \\
33 \pm 38 \\
6 \rightarrow 96 \\
38 \pm 40 \\
12 \rightarrow 103\end{array}$ & $\begin{aligned} 54 & \pm 38 \\
6 & \rightarrow 100 \\
56 & \pm 40 \\
354 & \rightarrow 154 \\
43 & \pm 40 \\
17 & \rightarrow 137\end{aligned}$ & $\begin{array}{l}0.61 \pm 0.20 \\
0.35 \rightarrow 0.90 \\
0.46 \pm 0.21 \\
0.17 \rightarrow 1.06 \\
0.46 \pm 0.22 \\
0.08 \rightarrow 0.88\end{array}$ \\
\hline $\begin{array}{l}-0.03 \mathrm{sec} . \\
\text { before QRS } \\
\text { termination }\end{array}$ & $\begin{array}{l}\text { (a) } \\
\text { (b) } \\
(c)\end{array}$ & $\begin{aligned} 217 & \pm 73^{\circ} \\
104 & \rightarrow 350 \\
156 & \pm 85 \\
6 & \rightarrow 296 \\
26 & \pm 104 \\
15 & \rightarrow 190\end{aligned}$ & $\begin{array}{c}200 \pm 37 \\
152 \rightarrow 267 \\
177 \pm 50 \\
86 \rightarrow 271 \\
173 \pm 47 \\
93 \rightarrow 261\end{array}$ & $\begin{array}{l}244 \pm 38 \\
182 \rightarrow 277 \\
258 \pm 47 \\
162 \rightarrow 358 \\
266 \pm 44 \\
179 \rightarrow 348\end{array}$ & $\begin{array}{l}0.39 \pm 0.21 \\
0.1 \mathrm{I} \rightarrow 0.60 \\
0.49 \pm 0.30 \\
0.1 \mathrm{I} \rightarrow \mathrm{I} \cdot 34 \\
0.41 \pm 0.22 \\
0.08 \rightarrow 0.98\end{array}$ \\
\hline $\begin{array}{l}\text { - } 0.02 \mathrm{sec} . \\
\text { before QRS } \\
\text { termination }\end{array}$ & $\begin{array}{l}(a) \\
(b) \\
(c)\end{array}$ & $\begin{array}{c}225 \pm 100 \\
58 \rightarrow 8 \\
197 \pm 85 \\
56 \rightarrow 3 \\
340 \pm 106 \\
178 \rightarrow 152\end{array}$ & $\begin{array}{c}238 \pm 76 \\
130 \rightarrow 23 \\
189 \pm 64 \\
60 \rightarrow 291 \\
183 \pm 67 \\
66 \rightarrow 326\end{array}$ & $\begin{array}{r}270 \pm 72 \\
171 \rightarrow 18 \\
239 \pm 70 \\
90 \rightarrow 25 \\
255 \pm 67 \\
99 \rightarrow 33\end{array}$ & $\begin{array}{l}0.17 \pm 0.12 \\
0.07 \rightarrow 0.29 \\
0.19 \pm 0.10 \\
0.03 \rightarrow 0.43 \\
0.18 \pm 0.10 \\
0.04 \rightarrow 0.42\end{array}$ \\
\hline $\begin{array}{l}\text { - o.or sec. } \\
\text { before QRS } \\
\text { termination }\end{array}$ & $\begin{array}{l}\text { (a) } \\
(b) \\
(c)\end{array}$ & $\begin{array}{c}10 \pm 85 \\
216 \rightarrow 115 \\
58 \pm 86 \\
16 \rightarrow 230 \\
64 \pm 96 \\
15 \rightarrow 220\end{array}$ & $\begin{aligned} 31 & \pm 64 \\
307 & \rightarrow 116 \\
54 & \pm 84 \\
8 & \rightarrow 210 \\
105 & \pm 91 \\
19 & \rightarrow 274\end{aligned}$ & $\begin{array}{c}65 \pm 64 \\
344 \rightarrow 157 \\
38 \pm 90 \\
13 \rightarrow 189 \\
320 \pm 106 \\
145 \rightarrow 121\end{array}$ & $\begin{array}{l}0.08 \pm 0.04 \\
0.04 \rightarrow 0.13 \\
0.07 \pm 0.04 \\
0.02 \pm 0.17 \\
0.07 \pm 0.03 \\
0.02 \rightarrow 0.14\end{array}$ \\
\hline
\end{tabular}

TABLE I2 Normal ranges of component amplitudes of $0.01,0.02,0.03$ vectors after $Q R S$ onset and before $Q R S$ terminations

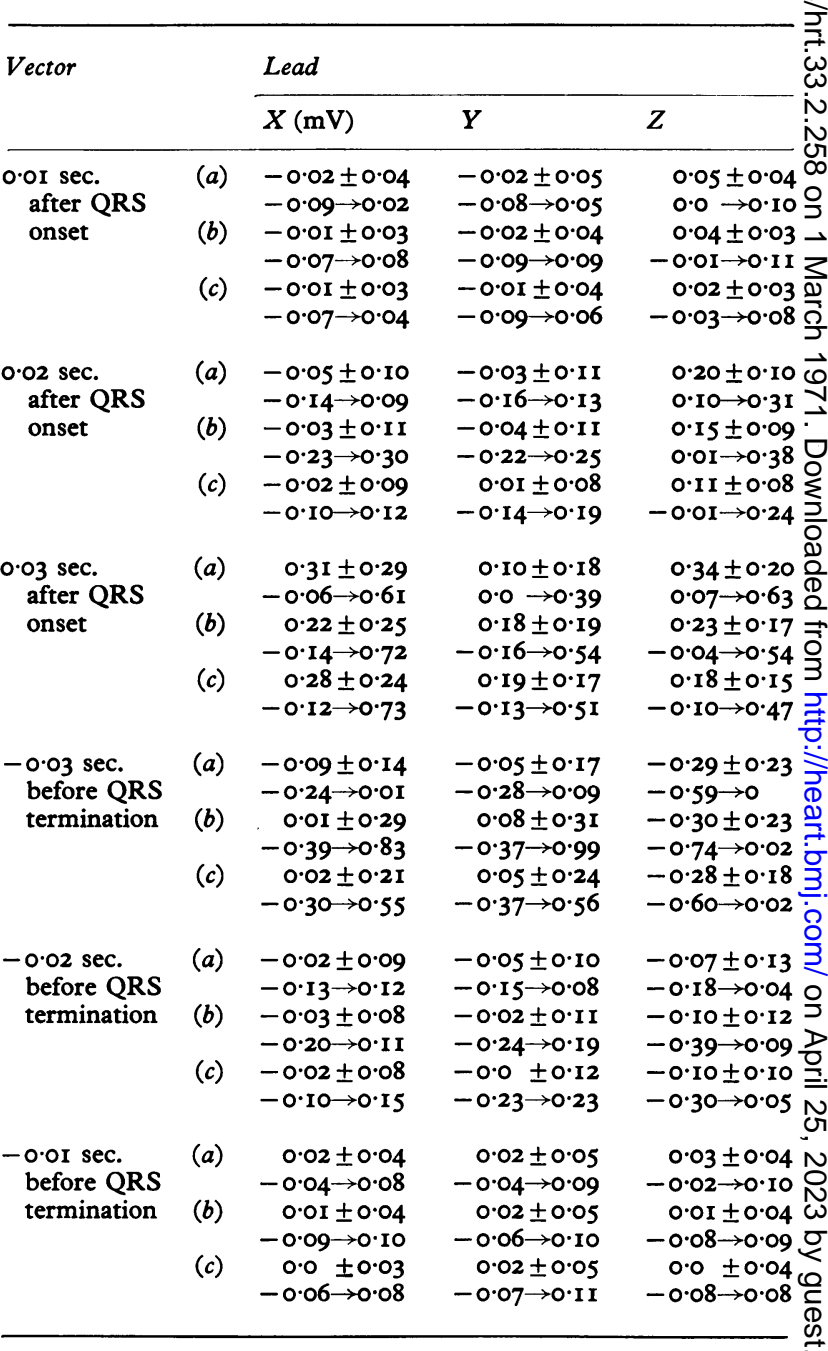


TABLE 13 Normal range of the time integrals $S A Q R S, S A T, S A S T$ (area under the $Q R S, T, S T$ waves, respectively). $S V G$, the spatial ventricular gradient, is obtained by summing other three time integrals

\begin{tabular}{|c|c|c|c|c|}
\hline \multirow[t]{2}{*}{ Vector } & & \multicolumn{3}{|l|}{ Lead } \\
\hline & & $X$ & $Y$ & $Z$ \\
\hline SA QRS & $\begin{array}{l}(a) \\
(b) \\
(c)\end{array}$ & $\begin{array}{c}23 \cdot 3 \pm 14.8 \mu \mathrm{V} \text { sec. } \\
8 \cdot 5 \rightarrow 49 \cdot 6 \\
23 \cdot 2 \pm 9 \cdot 9 \\
2 \cdot 2 \rightarrow 46 \cdot 2 \\
23 \cdot 9 \pm 9 \cdot 7 \\
9 \cdot 7 \rightarrow 42 \cdot 4\end{array}$ & $\begin{array}{r}25.3 \pm 10.8 \\
13.1 \rightarrow 45.0 \\
27.6 \pm 12.4 \\
4.9 \rightarrow 51.8 \\
24.5 \pm 12.6 \\
4.7 \rightarrow 48.4\end{array}$ & $\begin{aligned} &-2.3 \pm I I \cdot 4 \\
&-I 7.8 \rightarrow I 6.4 \\
&-3.5 \pm 9.8 \\
&-23.2 \rightarrow I 4.3 \\
&-4.2 \pm 8.2 \\
&-23.9 \rightarrow 9.9\end{aligned}$ \\
\hline SA $T$ & $\begin{array}{l}\text { (a) } \\
(b) \\
(c)\end{array}$ & $\begin{array}{r}37 \cdot 5 \pm 15.9 \\
21.6 \rightarrow 69.9 \\
30.4 \pm 14.9 \\
2.2 \rightarrow 62.9 \\
23.6 \pm 13.0 \\
1.4 \rightarrow 50.2\end{array}$ & $\begin{array}{r}25 \cdot 2 \pm 14 \cdot 7 \\
9 \cdot 7 \rightarrow 49 \cdot 8 \\
27 \cdot 3 \pm 14 \cdot 0 \\
4 \cdot 8 \rightarrow 62 \cdot 0 \\
23 \cdot 3 \pm 13 \cdot 2 \\
I \cdot I \rightarrow 48 \cdot 5\end{array}$ & $\begin{array}{r}I 4 \cdot I \pm I 4 \cdot 3 \\
I \cdot 3 \rightarrow 42 \cdot 6 \\
2 I \cdot 7 \pm 14 \cdot 8 \\
-2 \cdot 6 \rightarrow 52 \cdot 8 \\
I 8 \cdot 5 \pm 13 \cdot 0 \\
-2 \cdot 3 \rightarrow 44 \cdot 5\end{array}$ \\
\hline SA ST & $\begin{array}{l}\text { (a) } \\
(b) \\
(c)\end{array}$ & $\begin{array}{r}2 \cdot 2 \pm 4 \cdot 0 \\
-0.4 \rightarrow 4 \cdot 6 \\
1 \cdot 3 \pm 2 \cdot 2 \\
-2 \cdot 6 \rightarrow 5 \cdot 8 \\
0.4 \pm 1 \cdot 7 \\
-3 \cdot 3 \rightarrow 4 \cdot 3\end{array}$ & $\begin{array}{r}I \cdot 2 \pm I \cdot 2 \\
-0.4 \rightarrow 3.6 \\
I \cdot 5 \pm 3.0 \\
-5.0 \rightarrow I 0.7 \\
0.9 \pm I \cdot 7 \\
-I \cdot 8 \rightarrow 4.9\end{array}$ & $\begin{array}{r}2 \cdot 8 \pm 1 \cdot 5 \\
0.6 \rightarrow 4 \cdot 9 \\
2 \cdot 7 \pm 2 \cdot 3 \\
-0.7 \rightarrow 9 \cdot 1 \\
2 \cdot 2 \pm 2 \cdot 0 \\
-0.6 \rightarrow 7 \cdot 1\end{array}$ \\
\hline S VG & $\begin{array}{l}\text { (a) } \\
(b) \\
(c)\end{array}$ & $\begin{array}{l}52 \cdot 4 \pm 14 \cdot 7 \\
38 \cdot 1 \rightarrow 72 \cdot 1 \\
53 \cdot 5 \pm 18 \cdot 8 \\
18 \cdot 8 \rightarrow 87 \cdot 2 \\
47 \cdot 6 \pm 17 \cdot 6 \\
17 \cdot 3 \rightarrow 83 \cdot 3\end{array}$ & $\begin{array}{l}48 \cdot 4 \pm 19 \cdot 4 \\
30 \cdot 3 \rightarrow 75 \cdot 8 \\
55 \cdot 4 \pm 2 I \cdot 0 \\
I 9 \cdot 6 \rightarrow 97 \cdot 3 \\
48 \cdot 4 \pm 2 I \cdot 3 \\
I I \cdot 2 \rightarrow 9 I \cdot I\end{array}$ & $\begin{array}{r}14.6 \pm 17.8 \\
-7.5 \rightarrow 40 \cdot 6 \\
20.8 \pm 19.4 \\
-12.8 \rightarrow 64.9 \\
16.4 \pm 17.3 \\
-14.1 \rightarrow 49.5\end{array}$ \\
\hline
\end{tabular}

TABLE I4 Lead strengths of the modified and original axial lead system

\begin{tabular}{llll}
\hline & $X$ & $Y$ & $Z$ \\
\hline Axial & $\mathrm{I} \cdot 6 \mathrm{I}$ & $\mathrm{I} \cdot 48$ & $2 \cdot 24 \mathrm{mV} / \mathrm{cm}$. \\
Modified axial & $\mathrm{I} \cdot 48$ & $\mathrm{I} \cdot 48$ & $\mathrm{I} \cdot 48$ \\
\hline
\end{tabular}

